\title{
Spin Manipulation and Relaxation in Spin-Orbit Qubits
}

\author{
Massoud Borhani ${ }^{1,2}$ and Xuedong $\mathrm{Hu}^{1}$ \\ ${ }^{1}$ Department of Physics, University at Buffalo, SUNY, Buffalo, NY 14260-1500, USA \\ ${ }^{2}$ Laboratory for Physical Sciences, 8050 Greenmead Drive, College Park, MD 20740, USA
}

(Dated: October 21, 2019)

\begin{abstract}
We derive a generalized form of the Electric Dipole Spin Resonance (EDSR) Hamiltonian in the presence of the spin-orbit interaction for single spins in an elliptic quantum dot (QD) subject to an arbitrary (in both direction and magnitude) applied magnetic field. We predict a nonlinear behavior of the Rabi frequency as a function of the magnetic field for sufficiently large Zeeman energies, and present a microscopic expression for the anisotropic electron g-tensor. Similarly, an EDSR Hamiltonian is devised for two spins confined in a double quantum dot (DQD), where coherent Rabi oscillations between the singlet and triplet states are induced by jittering the interdot distance at the resonance frequency. Finally, we calculate two-electron-spin relaxation rates due to phonon emission, for both in-plane and perpendicular magnetic fields. Our results have immediate applications to current EDSR experiments on nanowire QDs, g-factor optimization of confined carriers, and spin decay measurements in DQD spin-orbit qubits.
\end{abstract}

PACS numbers:

An electron spin confined in a semiconductor quantum dot is a promising candidate for spin-based quantum information processing $\frac{112}{2}$. To implement reliable quantum gates and quantum registers, a complete theoretical knowledge of all single- and two-spin-qubit decay channels is essential in order to mitigate the decoherence and relaxation of qubits. Spin-orbit interaction is one of the most important mechanisms of spin mixing of different orbital states in semiconductor structures. When combined with phonons or charge fluctuations, this spin mixture results in a variety of spin decay channels, although they have all turned out to be quite weak for confined electrons ${ }^{3}{ }^{5}$, leading to impressively long single-spin relaxation time $\left(T_{1}\right)$ (of the order of seconds at 1 Tesla in GaAs and minutes in $\mathrm{Si}: \mathrm{P}$ )617. The dominant electron spin decoherence channel for GaAs QD and Si donors is pure dephasing due to hyperfine interaction with the environmental nuclear spins 89 , with the corresponding coherence time $\left(T_{2}\right)$ having been pushed to few hundreds of microseconds in $\mathrm{GaAs}^{8}$ and a few seconds in $\mathrm{Si}: \mathrm{P} 7$. This significant separation of $T_{1}$ and $T_{2}$ time scales means that spin-orbit interaction can play a constructive role in qubits with a relatively strong spin-orbit interaction.

Exploiting the orbital part of the electrons and holes to manipulate their spins (via the spin-orbit coupling) is becoming a common practice in recent experimental and theoretical works on extended and confined electrons in heterostructures with strong spin-orbit interaction 2110 15. GigaHertz manipulation of confined electrons in semiconductor QDs is achievable, in the presence of an applied magnetic field, accompanied by an ac electric field 12 . For small Zeeman splitting (compared to the orbital excitations), the Rabi frequency shows a linear dependence on both applied magnetic and electric fields. This spinelectric-field coupling was observed in transport experiments on lateral GaAs QDs and InAs nanowires in the spin-blockade regime ${ }^{13} \mid 15$, where the Rabi spin-flip time of $10 \mathrm{~ns}$ has been reported. Such a fast spin rotation is a key step towards realizing single- and two-qubit gates for localized spin qubits 12 .

In this paper, we first present the general EDSR Hamiltonian for a single electron spin confined in an elliptic 2D $\mathrm{QD}$, in the presence of an arbitrarily large magnetic field. The study of elliptic confinement is crucial in nano-wire QDs, where the electron orbital wave function is strongly squeezed in two directions $16 / 17$. In contrast to previous works 1112 , we show that the Rabi frequency exhibits a pronounced non-linear behavior as a function of the applied magnetic field for sufficiently large QDs and Zeeman energies. We also calculate the second-order corrections of the spin-orbit coupling to the EDSR Hamiltonian, and provide an analytical microscopic expression for the g-factor renormalization. The ability to engineer the g-factor of a confined electron is essential for individual addressing of spin-orbit qubits via electric pulses $14 \mid 15$. In addition, we propose a scheme to perform coherent rotation in the singlet-triple subspace of two electron spins confined in a double QD. Again, Zeeman energy and the spin-orbit interaction play the central role in the electrical manipulation of the two-spin states, where the corresponding Rabi frequency is linear, in the leading order, in magnetic field and the spin-oribt coupling. The proposed two-electron EDSR offers an alternative way to implement coherent swap gates in coupled spin-orbit qubits, without a magnetic field gradient. Finally, we calculate the phonon-induced spin relaxation time of the two-spin system and find that the resulting time scales are relatively long, which justifies the efficiency of our two-spin EDSR scheme.

The effective EDSR Hamiltonian for an elliptic dot. Consider a single electron in an elliptic 2D quantum dot, with confining frequencies $\omega_{x}$ and $\omega_{y}$, subject to an applied magnetic field $\boldsymbol{B}=B(\sin \theta \cos \phi, \sin \theta \sin \phi, \cos \theta)$. 
TABLE I: The $\left\{\alpha_{i}, \tilde{\alpha}_{i}\right\}$ coefficients of the transformation matrix $\mathcal{S}$ in Eqs. (5) 7 as a function of the Zeeman interaction, the spin-orbit lengths, the cyclotron frequency $\omega_{c}=e B_{z} / m c$, and the lateral confining frequencies. Note that $\mathcal{M}$ is a common factor which appears in all coefficients. Similarly, $\left\{\beta_{i}, \tilde{\beta}_{i}\right\}$ are given by the following substitutions: $\beta_{1}=\alpha_{1}\left(\omega_{x} \rightarrow\right.$ $\left.\omega_{y}, \lambda_{-} \rightarrow \lambda_{+}\right), \beta_{2}=-\alpha_{2}\left(\omega_{x} \rightarrow \omega_{y}, \lambda_{-} \rightarrow \lambda_{+}\right), \tilde{\beta}_{1}=-\tilde{\alpha}_{1}\left(\lambda_{-} \rightarrow \lambda_{+}\right)$, and $\tilde{\beta}_{2}=\tilde{\alpha}_{2}\left(\omega_{x} \rightarrow \omega_{y}, \lambda_{-} \rightarrow \lambda_{+}\right)$; For example, $\beta_{2}=\hbar E_{Z}\left(\hbar \omega_{c}\right)\left(\hbar \omega_{y}\right)^{2} / m \mathcal{M} \lambda_{+}$.

\begin{tabular}{|c||c|c|c|c|}
\hline $\mathcal{M}$ & $m \mathcal{M} \lambda_{-} \alpha_{1} / \hbar$ & $\mathcal{M} \lambda_{-} \alpha_{2}$ & $m \mathcal{M} \lambda_{-} \tilde{\alpha}_{1} / \hbar$ & $\mathcal{M} \lambda_{-} \tilde{\alpha}_{2}$ \\
\hline$E_{Z}^{2}\left(\hbar \omega_{c}\right)^{2}-\left[\left(\hbar \omega_{x}\right)^{2}-E_{Z}^{2}\right]\left[\left(\hbar \omega_{y}\right)^{2}-E_{Z}^{2}\right]$ & $E_{Z}\left[\left(\hbar \omega_{x}\right)^{2}-E_{Z}^{2}\right]$ & $-E_{Z}\left(\hbar \omega_{c}\right)\left(\hbar \omega_{x}\right)^{2}$ & $E_{Z}^{2}\left(\hbar \omega_{c}\right)$ & $E_{Z}^{2}\left[\left(\hbar \omega_{x}\right)^{2}+\left(\hbar \omega_{c}\right)^{2}-E_{Z}^{2}\right]$ \\
\hline
\end{tabular}

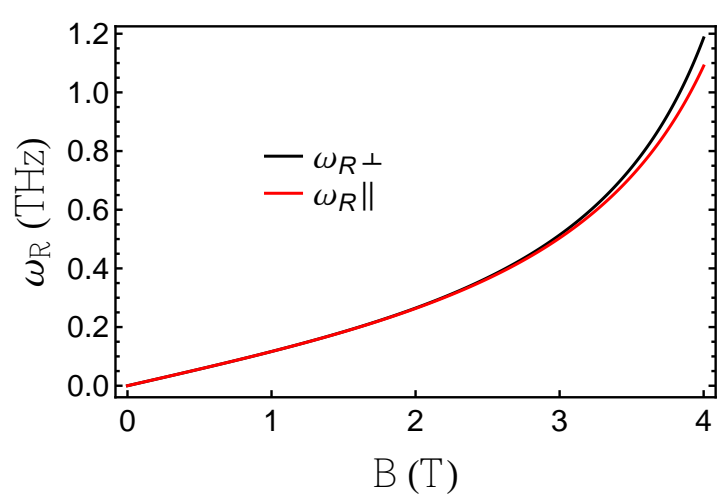

FIG. 1: (color online). The Rabi frequency versus the magnetic field for an electrically driven single spin in a typical InAs nanowire QD ${ }^{16}$ : $\omega_{x}=3 \mathrm{meV}, \omega_{y}=40 \mathrm{meV}$, and $\lambda_{+} \sim 100 \mathrm{~nm}$, for both perpendicular $\left(\omega_{R \perp}\right)$ and in-plane $\left(\omega_{R \|}\right)$ magnetic fields. The electric field is in-plane and along the $x$ axis. The non-linear dependence shows up for $B$ fields above $2 \mathrm{~T}$, although this threshold can be substantially reduced for larger dots and/or for materials with stronger spinorbit interaction.

The Hamiltonian for the electron is

$$
\begin{aligned}
H & =H_{d}+H_{Z}+H_{s o}, \\
H_{d} & =\frac{p^{2}}{2 m}+\frac{1}{2} m\left(\omega_{x}^{2} x^{2}+\omega_{y}^{2} y^{2}\right), \\
H_{Z} & =\frac{1}{2} g \mu_{B} \boldsymbol{B} \cdot \boldsymbol{\sigma}=\frac{1}{2} E_{Z} \boldsymbol{n} \cdot \boldsymbol{\sigma} \\
H_{s o} & =\mathcal{K} \cdot \boldsymbol{\sigma}, \quad \mathcal{K} \equiv \frac{\hbar}{m}\left(p_{y} / \lambda_{-}, p_{x} / \lambda_{+}, 0\right),
\end{aligned}
$$

where $m$ is the effective mass of the electron, $\boldsymbol{p}=$ $-i \hbar \partial / \partial \boldsymbol{r}+(e / c) \boldsymbol{A}(\boldsymbol{r})$ the electron kinetic momentum in two dimensions, and $c$ the speed of light in vacuum. $\lambda_{ \pm}=\hbar / m(\beta \pm \alpha)$ are the effective spin-orbit lengths in terms of the linear Rashba $(\alpha)$ and Dresselhaus $(\beta)$ coupling constants 18119 . We restrict our consideration to quantum dots with strong confinement along one axis ([001]), such as, e.g., quantum dots defined in a twodimensional electron gas (2DEG). With a uniform $\boldsymbol{B}$ field, the vector potential is $\boldsymbol{A}(\boldsymbol{r})=B_{z}(-y / 2, x / 2,0)$ in the symmetric gauge. With the motion along $z$ strongly quantized (the 2DEG thickness $\mathcal{D} \ll \sqrt{\hbar c / e B_{x(y)}}$ ), the in-plane components $B_{x}$ and $B_{y}$ are not included in $\boldsymbol{A}(\boldsymbol{r})$. The magnetic field also induces a Zeeman splitting $E_{Z}=g \mu_{B} B$, with a spin quantization axis $\boldsymbol{n}=\boldsymbol{B} / B$. Note that the linear-in-momentum spin-orbit interaction is given in a coordinate system which is rotated by $\pi / 4$ along the $z$ axis with respect to the crystallographic axes of the crystal and it is the sum of both Rashba and Dresselhaus terms.

In order to diagonalize the Hamiltonian in Eq. (1), we invoke the Schrieffer-Wolff transformation $H \rightarrow$ $e^{-\mathcal{S}} H e^{\mathcal{S} 5120}$, and remove the spin-oribit interaction in the leading order by imposing $\left[H_{d}+H_{Z}, \mathcal{S}\right]=H_{s o}$. The resulting $\mathcal{S}$ matrix is presented here to all orders in the Zeeman interaction, but in the first order in spin-orbit coupling: $\mathcal{S}=\mathcal{S}^{(0)}+\mathcal{S}^{(1)}+\mathcal{S}^{(2)}$,

$$
\begin{aligned}
\mathcal{S}^{(0)} & =i \boldsymbol{\xi} \cdot \boldsymbol{\sigma}, \\
\mathcal{S}^{(1)} & =i[\boldsymbol{n} \times \boldsymbol{\eta}] \cdot \boldsymbol{\sigma}, \\
\mathcal{S}^{(2)} & =i[\boldsymbol{n} \times(\boldsymbol{n} \times \boldsymbol{\gamma})] \cdot \boldsymbol{\sigma}, \\
\boldsymbol{\xi} & =\left(y / \lambda_{-}, x / \lambda_{+}, 0\right), \\
\boldsymbol{\eta} & =\left(\alpha_{1} p_{y}+\alpha_{2} x, \beta_{1} p_{x}+\beta_{2} y, 0\right), \\
\boldsymbol{\gamma} & =\left(\tilde{\alpha}_{1} p_{x}+\tilde{\alpha}_{2} y, \tilde{\beta}_{1} p_{y}+\tilde{\beta}_{2} x, 0\right) .
\end{aligned}
$$

The coefficients $\left\{\alpha_{i}, \beta_{i}, \tilde{\alpha}_{i}, \tilde{\beta}_{i}\right\}$ are given in Table I. Due to the spin-orbit interaction, the spin and orbital part of the rotated states are strongly coupled to each other, which opens up the possibility of spin manipulation via driving by an $a c$ electric field $V(\boldsymbol{r}, t)=e \boldsymbol{E} \cdot \boldsymbol{r}$ (within the dipole approximation). The effective Hamiltonian, $H_{E D S R}=H_{Z}+\left\langle\psi_{0}|[S, V(\boldsymbol{r}, t)]| \psi_{0}\right\rangle$ for the spin sector of an electron confined in the ground orbital state of an elliptic dot, is obtained by averaging over the orbital ground state

$$
\begin{aligned}
H_{E D S R} & =H_{Z}+\frac{1}{2} \boldsymbol{h}(t) \cdot \boldsymbol{\sigma} \\
\boldsymbol{h}(t) & =2 \boldsymbol{n} \times(\mathcal{F}+\mathcal{G}) \\
\mathcal{F} & =e \hbar\left(\alpha_{1} E_{y}, \beta_{1} E_{x}, 0\right) \\
\mathcal{G} & =e \hbar\left(-n_{z} \tilde{\beta}_{1} E_{y}, n_{z} \tilde{\alpha}_{1} E_{x}, n_{x} \tilde{\beta}_{1} E_{y}-n_{y} \tilde{\alpha}_{1} E_{x}\right) .
\end{aligned}
$$


We note that the effective ac magnetic field $\boldsymbol{h}(t)$ vanishes in the absence of the applied magnetic field, and is always perpendicular to $\boldsymbol{B}$. This is a distinct feature of the linear spin-orbit interaction in the leading order. Moreover, for an in-plane magnetic field, the vector $\mathcal{G}$ is identically zero because $\omega_{c}=0$ (see Table I).

In general, Rabi frequency is strongly dependent on the directions of the magnetic and the $a c$ electric fields, the confinement frequencies, and the orientation of the QD with respect to the crystallographic axes. For small Zeeman energies $\left(E_{Z} \ll \hbar \omega_{x}, \hbar \omega_{y}\right)$, the Rabi frequency is linear in $E_{Z}$ 12. In Fig. 1, we have plotted the Rabi frequency $\omega_{R}=\max |\boldsymbol{h}(t)| / \hbar$, between the Zeeman sublevels, as a function of the applied $B$ field. A clear nonlinear behavior arises for magnetic fields above $2 \mathrm{~T}$. To obtain these results, we used parameters for a typical InAs nanowire QD. This nonlinearity is a direct consequence of the exact treatment of the Zeeman interaction in calculating the Schrieffer-Wolff matrix $\mathcal{S}$.

The above consideration is restricted to a twodimensional QD where the effective spin-orbit interaction is linear in momentum. In reality, all QDs have finite thickness $\mathcal{D}$ of the order of a few nanometers. Therefore, the cubic Dresselhaus term $H_{s o}^{3 D} \sim$ $\frac{\beta \mathcal{D}^{2}}{\hbar^{2}}\left(p_{y} p_{x} p_{y} \sigma_{x}-p_{x} p_{y} p_{x} \sigma_{y}\right)$ can also, in principle, induce spin transitions $\frac{18}{}$, though in the leading order it is ineffective in the presence of harmonic confinements and in-plane magnetic fields 12 .

For spin manipulations in QDs, a comprehensive knowledge of the electron g-tensor is crucia 1 14115. We have calculated the second-order corrections of the linear spin-orbit interaction to the effective Hamiltonian, Eq. (8), which lead to the renormalization of the gfactor ${ }^{12121}$. For an in-plane magnetic field along the $x$ axis, the renormalized g-tensor $\tilde{g}_{i j}$ of an electron in the ground state of the harmonic potential reads (results for an arbitrary $B$ field direction is presented in appendix A)

$$
\begin{aligned}
& \tilde{g}_{x x}=g_{x x}\left(1-\frac{\hbar^{3} \omega_{x}}{m \lambda_{+}^{2}\left[\left(\hbar \omega_{x}\right)^{2}-E_{Z}^{2}\right]}\right), \\
& \tilde{g}_{y y}=g_{y y}=\tilde{g}_{z z}=g_{z z}=g_{x x}=g .
\end{aligned}
$$

This renormalization of the g-factor can be used to address nearby spins selectively, and has immediate applications in current EDSR experiments on confined carriers $14 \mid 15$.

Two-electron spin Hamiltonian. We now consider two electrons confined in a DQD separated by $2 d$, as shown in Fig. 2, and the vector $\boldsymbol{d}$ denotes the orientation of the DQD with respect to the crystallographic axes. This setup has been the cornerstone of recent experimental works on spin-selective transport, where the lifetime of different spin states are examined through the spinblockade effect $13-15|22| 23$. In the absence of the spin-orbit interaction, the low energy subspace of the two-electron Hilbert space consists of the following four states

$$
\left\{\Phi_{i}\right\}_{i=1, \ldots, 4}=\left\{\Psi_{+} S, \Psi_{-} T_{+}, \Psi_{-} T_{0}, \Psi_{-} T_{-}\right\}
$$



FIG. 2: (color online). Schematic of a singlet-triplet DQD spin qubit ${ }^{22}$ in the presence of an in-plane magnetic field and an $a c$ electric field. The distance between the center of the double elliptic dots is $2 d$ and the driving electric field is applied along the DQD in order to induce maximum electric dipole moment. In the upper right, the corresponding energy levels of two-spins has been shown for the regime $J \ll E_{Z}$.

where $S$ and $T_{ \pm, 0}$ refer to the spin singlet and triplet states, respectively, and $\Psi_{ \pm}$are their corresponding symmetric and anti-symmetric orbital wavefunctions. By including the spin-orbit coupling, the singlet and triplet states are coupled provided that an external magnetic field is applied. For small magnetic fields, the effective Hamiltonian for the two spin confined in a double dot is given by 24

$$
\begin{aligned}
H_{e x}= & (J / 4) \boldsymbol{\sigma}_{1} \cdot \boldsymbol{\sigma}_{2}+\mu \boldsymbol{B} \cdot\left(\boldsymbol{\sigma}_{1}+\boldsymbol{\sigma}_{2}\right) \\
& +\boldsymbol{a} \cdot\left(\boldsymbol{\sigma}_{1}-\boldsymbol{\sigma}_{2}\right)+\boldsymbol{b} \cdot\left(\boldsymbol{\sigma}_{1} \times \boldsymbol{\sigma}_{2}\right)
\end{aligned}
$$

where $\mu \equiv g \mu_{B} / 2$ and $J$ is the singlet-triplet exchange splitting. Here we keep only the leading order terms in spin-orbit coupling and neglect the second and higher order terms. The only linear terms in spin-orbit (and Zeeman) interaction are the following vectors

$$
\begin{aligned}
\boldsymbol{a} & \equiv-\mu \boldsymbol{B} \times \operatorname{Re}\left\langle\Psi_{+}|\boldsymbol{\xi}| \Psi_{-}\right\rangle, \\
\boldsymbol{b} & \equiv-\mu \boldsymbol{B} \times \operatorname{Im}\left\langle\Psi_{+}|\boldsymbol{\xi}| \Psi_{-}\right\rangle,
\end{aligned}
$$

where $a=|\boldsymbol{a}|$ and $b=|\boldsymbol{b}|$ denote the strength of the spin-orbit matrix elements. Note that the Hamiltonian in Eq. (12) has the rotated spin-orbit states $\left\{U^{0} \Phi_{i}\right\}$, where $U^{0}=\exp \left[-\mathcal{S}^{(0)}{ }^{24}\right.$ as the basis. For an in-plane magnetic field, $S$ and $T_{0}$ are coupled via the $z$ component of the vector $\boldsymbol{a}$ (there is no coupling, in the leading order, between these two states for a perpendicular magnetic field). Specifically, $a_{z}=\frac{\mu B d}{2 \lambda_{+}}$(in the Heitler-London approximation), provided that $\boldsymbol{B}$ and $\boldsymbol{d}$ are along the $x$ axis. Rabi Oscillations between the singlet and the triplet states have already been observed in DQDs using the hyperfine field gradient ${ }^{22}$. When spin-orbit interaction is included in the consideration, a time-dependent $a_{z}$ can also induce coherent oscillations between $S$ and $T_{0}$ states, providing an alternative way to manipulate the singlet and triplet states. For example, a sinusoidal electric field pulse can change the inter-dot distance periodically to form a breathing $\mathrm{DQD}, d=d_{0}+\delta \sin (\omega t)$. The corresponding Rabi frequency is then given by $\omega_{R}=\frac{\mu B \delta}{2 \hbar \lambda_{+}}$, 


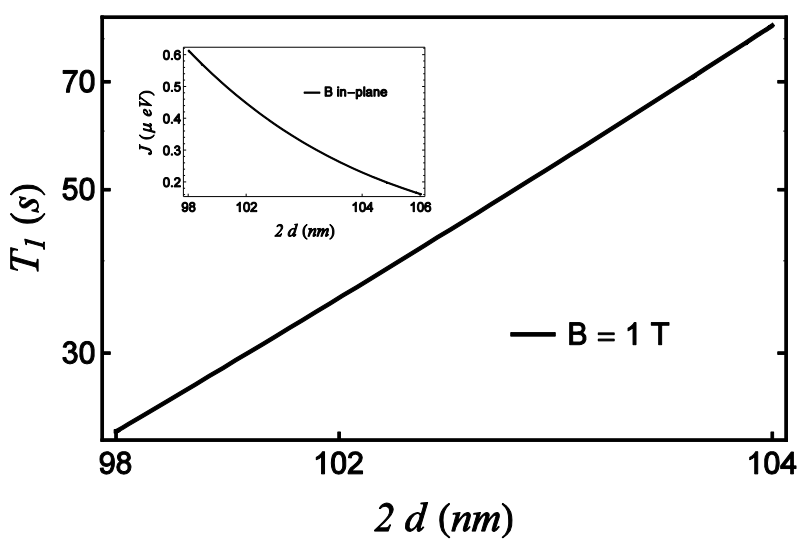

FIG. 3: The logarithmic plot of the triplet-singlet $\left(T_{0}-S\right)$ relaxation time, for a DQD along the $x$ axis, as a function of the distance between the two QDs. The applied magnetic field $B=1 T$ ( in this case, the rate has quadratic dependence on the field). The inset shows the corresponding dependence of $J$ on $2 d$, calculated in the Heitler-London approximation. The material parameters are chosen for GaAs quantum dots with $\lambda_{+} \sim 5 \mu \mathrm{m}, l=22 \mathrm{~nm}, e_{14}=1.4 \times 10^{9} \mathrm{~V} / \mathrm{m}, \kappa=13.1$, $\rho=5.3 \times 10^{3} \mathrm{~kg} / \mathrm{m}^{3}$, and $s_{t}=2.5 \times 10^{3} \mathrm{~m} / \mathrm{s}$.

where $\delta \ll d_{0}$ is the amplitude of the breathing DQD, and $\delta$ is linearly proportional to the applied $a c$ electric field $E$. For InAs QDs, where the g-factor $g \approx 10$ and $\lambda_{+} \approx 100 \mathrm{~nm}$, a $\delta \approx 1 \mathrm{~nm}$ at $B=1 \mathrm{~T}$ would allow a $\pi / 2$ pulse on the order of $1 \mathrm{~ns}$.

The exchange coupling between the two spins also oscillates as we periodically change their relative distance. Geometrically, this leads to a breathing Bloch sphere which beats at the driving frequency. However, if the amplitude of modulations is reasonably small, they yield small deviations $\delta J$ from the equilibrium value $J$ and the coherent Rabi oscillations should still be observable in the leading order of the spin-orbit interaction ${ }^{12}$.

Singlet-triplet relaxation rates. Due to the spin mixing via the spin-orbit interaction, phonon emission can lead to relaxation (and/or leakage out) of the two-spin singlet and triplet states. In III-V semiconductors like GaAs or InAs, piezoelectric coupling to acoustic phonons is the dominant relaxation channe $\frac{425}{4}$. With an in-plane magnetic field (in which regime $S-T_{+}$and $T_{0}-T_{+}$do not couple in the leading order) and in the leading order of the spin-orbit coupling, the triplet-singlet relaxation rate is given by

$$
\begin{aligned}
\Gamma_{T_{0} \rightarrow S} & =\frac{4\left(e e_{14}\right)^{2}}{\pi \kappa^{2} \rho \hbar^{2} s_{t}^{3}} \cdot\left(n_{q_{0}}+1\right) e^{-4 d^{2} / l^{2}} \cdot \frac{a_{z}^{2}}{J} f(u), \\
a_{z} & =\frac{\mu}{2}\left(\frac{B_{x} d_{x}}{\lambda_{+}}-\frac{B_{y} d_{y}}{\lambda_{-}}\right) \\
f(u) & =\frac{21 u-2 u^{3}+\left[4 u^{2}\left(u^{2}-3\right)-21\right] D(u)}{2 u^{5}} \\
D(u) & =e^{-u^{2}} \int_{0}^{u} e^{v^{2}} d v, \quad u \equiv \frac{l J}{\sqrt{2} \hbar s_{t}},
\end{aligned}
$$

where $e_{14}$ and $\kappa$ are the piezoelectric and the dielectric constants of the material, respectively, $\rho$ is the density of the sample, $s_{t}$ is the speed of transverse acoustic phonons, and $l$ is the size of each quantum dot. This relaxation rate is valid when $a_{z} \ll J$. Moreover, $a_{z}$ has a geometrical structure which enables us to mitigate this relaxation channel by carefully choosing the direction of the magnetic field with respect to the crystallographic axes and the DQD orientation. In Fig. 3, we have plotted the relaxation rate in Eq. (15), together with the exchange splitting (within the Heitler-London approximation), as a function of the DQD separation $2 d$ in the presence of an applied in-plane magnetic field of $1 \mathrm{~T}$. The resulting time scales are relatively long for small Zeeman energies (below 1 Tesla). Therefore, we conclude that in this regime single-spin and/or hyperfine-induced triplet-singlet relaxations dominate the spin relaxation processes, while dephasing is probably dominated by hyperfine interaction ${ }^{9}$, charge noise ${ }^{26}$, and phonons $s^{27}$. Note that while the Hamiltonian in Eq. (12) is quite generic for any inter-dot coupling, the analytical results presented here are for a relatively narrow inter-dot distance regime, because our analytical calculation is bound from the small $d$ by the validity of our Heitler-London model to calculate $J$ and $a_{z}$, and from the large $d$ by the requirement that singlet and triplet states are close to the two-electron eigenstates.

For a perpendicular magnetic field, the only states which are coupled, in the leading order of the spin-orbit interaction, are $S$ and $T_{+}$(See appendix B). Although there is no analytical form for this relaxation rate, we have found numerical values showing very slow leakage rate $\left(0.001 \mathrm{~s}^{-1}\right.$ or slower when $\left.a, b \ll E_{Z}-J\right)$ for magnetic fields of interest between $0.1-1 \mathrm{~T}$.

In conclusion, we have derived the most general form of the EDSR Hamiltonian for an elliptic quantum dot, in the presence of the (linear) spin-orbit interaction. We find a nonlinear $B$-field dependence for the Rabi frequency of the resulting single-spin EDSR. We also propose electrical manipulation of two-electron-spin states via inter-dot modulation. We find that the phonon-induced tripletsinglet relaxation rates are typically small, so that coherent Rabi oscillations can, in principle, be observed with current experimental setups in DQD spin-orbit qubits.

We thank financial support from NSA/LPS through US ARO, and DARPA QuEST through AFOSR. 


\section{Appendix A: The renormalized g-tensor for confined electrons}

In this subsection, we calculate the renormalization of the confined electron g-factor in the presence of an applied magnetic field. Through the Schrieffer-Wolff transformation, the effective Hamiltonian (up to second order in spin-orbit interaction and to all orders in Zeeman coupling) is $[5,12]$

$$
\begin{aligned}
H_{e f f} & =H_{d}+H_{Z}+\Delta H, \\
\Delta H & =\frac{1}{2}\left[S, H_{s o}\right],
\end{aligned}
$$

where the transformation matrix $S$ is introduced in the main text of the paper. By calculating the above commutator, one can obtain the (second-order) corrections to the Hamiltonian and the electron spin g-tensor. The resulting Hamiltonian has a rather complicated form, thus we only present two special cases of particular interest:

$\Delta H_{\|}=-\frac{\hbar^{2}}{2 m}\left(\frac{1}{\lambda_{+}^{2}}+\frac{1}{\lambda_{-}^{2}}\right)+\frac{\hbar^{2} \tilde{\beta}_{2}}{2 m \lambda_{+}}+\frac{\hbar \beta_{1}}{m \lambda_{+}} p_{x}^{2} \sigma_{x}-\frac{\hbar}{2 m \lambda_{-}}\left\{p_{x}, p_{y}\right\} \sigma_{y}+\left[\frac{\hbar}{m \lambda_{+} \lambda_{-}}\left(x p_{y}-y p_{x}\right)-\frac{\hbar \tilde{\beta}_{2}}{m \lambda_{-}} x p_{y}\right] \sigma_{z}$

where $\{U, V\} \equiv U V+V U$ is the anticommutator of $U$ and $V$. In an in-plane $B$ field, generally all components of the g-tensor is renormalized, depending on the electron orbital state. However, for an electron in the ground state, the quantum mechanical average of the orbital operators multiplying $\sigma_{y}$ and $\sigma_{z}$ vanish, and only $\tilde{g}_{x x}$ differs from the bulk g-factor.

2. B perpendicular to the $x-y$ plane

$$
\begin{aligned}
\Delta H_{\perp}= & -\frac{\hbar^{2}}{2 m}\left(\frac{1}{\lambda_{+}^{2}}+\frac{1}{\lambda_{-}^{2}}+\frac{\alpha_{2}}{\lambda_{+}}-\frac{\beta_{2}}{\lambda_{-}}-\frac{\tilde{\alpha}_{2}}{\lambda_{-}}-\frac{\tilde{\beta}_{2}}{\lambda_{+}}\right)-\frac{\hbar^{2} \omega_{c}}{2}\left(\frac{\alpha_{1}}{\lambda_{+}}+\frac{\beta_{1}}{\lambda_{-}}+\frac{\tilde{\alpha}_{1}}{\lambda_{-}}-\frac{\tilde{\beta}_{1}}{\lambda_{+}}\right) \\
& +\frac{\hbar}{m}\left[\frac{1}{\lambda_{+} \lambda_{-}}\left(x p_{y}-y p_{x}\right)+\frac{1}{\lambda_{+}}\left(\tilde{\alpha}_{1}+\beta_{1}\right) p_{x}^{2}+\frac{1}{\lambda_{-}}\left(\alpha_{1}-\tilde{\beta}_{1}\right) p_{y}^{2}+\frac{1}{\lambda_{-}}\left(\alpha_{2}-\tilde{\beta}_{2}\right) x p_{y}+\frac{1}{\lambda_{+}}\left(\tilde{\alpha}_{2}+\beta_{2}\right) y p_{x}\right] \sigma_{z}
\end{aligned}
$$

In this case, only $g_{z z}$ is renormalized, for any electronic orbital state.

\section{Appendix B: The leakage rate out of the singlet-triplet subspace}

Here we calculate the two-electron spin relaxation rate for a double dot in a perpendicular magnetic field, for the regime where $a, b \ll E_{Z}-J$. In a perpendicular $B$ field, there is no coupling between $S-T_{0}$, in the leading order of the spin-orbit interaction. The only leakage channel is $S \rightarrow T_{+}$[see Eq. (12) in the text], 


$$
\begin{aligned}
\Gamma_{S \rightarrow T_{+}} & =\frac{8\left(e e_{14}\right)^{2}}{\pi \kappa^{2} \rho \hbar^{2} s_{t}^{3}}\left(n_{q_{0}}+1\right) \cdot e^{-\frac{d^{2}\left(l^{4}+4 l_{B}^{4}\right)}{l^{2} l_{B}^{4}}} \cdot \frac{\left(a_{x}-b_{y}\right)^{2}+\left(a_{y}-b_{x}\right)^{2}}{E_{Z}-J} \cdot\left\{g\left(d, q_{0}\right)+\frac{f(r)}{8}\right\}, \\
\boldsymbol{a} & =\frac{\mu B_{z}}{2}\left(-\frac{d_{x}}{\lambda_{+}} \hat{x}+\frac{d_{y}}{\lambda_{-}} \hat{y}\right), \\
\boldsymbol{b} & =\frac{\mu B_{z} l^{2}}{4 l_{B}^{2}}\left(\frac{d_{y}}{\lambda_{+}} \hat{x}-\frac{d_{x}}{\lambda_{-}} \hat{y}\right), \\
g\left(d, q_{0}\right) & =\int_{0}^{\pi} d \theta \sin ^{3} \theta \cos ^{2} \theta e^{-l^{2} q_{0}^{2} \sin ^{2} \theta / 2} I_{0}\left(\frac{2 l^{2} d q_{0}}{l_{B}^{2}} \sin \theta\right) \\
& +\frac{l_{B}^{4}}{4 l^{4} d^{2} q_{0}^{2}} \int_{0}^{\pi} d \theta \sin ^{3} \theta e^{-l^{2} q_{0}^{2} \sin ^{2} \theta / 2}\left\{I_{2}\left(\frac{2 l^{2} d q_{0}}{l_{B}^{2}} \sin \theta\right)+\frac{2 l^{2} d q_{0}}{l_{B}^{2}} \sin \theta \cdot I_{3}\left(\frac{2 l^{2} d q_{0}}{l_{B}^{2}} \sin \theta\right)\right\},
\end{aligned}
$$

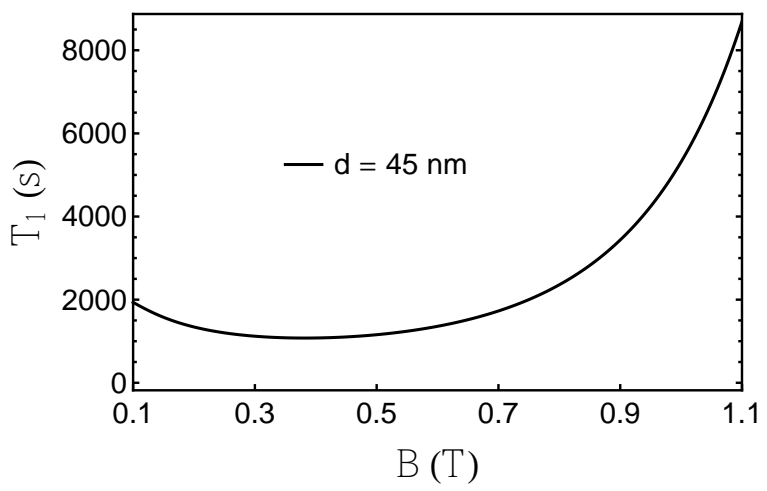

FIG. 4: The singlet-triplet $\left(S \rightarrow T_{+}\right)$relaxation time, for a DQD along the $x$ axis, as a function of applied (perpendicular) magnetic field. The material parameters are chosen for GaAs quantum dots with $\lambda_{s o}=5 \mu \mathrm{m}, l=30 \mathrm{~nm}$ (at $B=0$ ), $e_{14}=1.4 \times 10^{9} \mathrm{~V} / \mathrm{m}, \kappa=13.1, \rho=5.3 \times 10^{3} \mathrm{~kg} / \mathrm{m}^{3}$, and $s_{t}=2.5 \times 10^{3} \mathrm{~m} / \mathrm{s}$. where $q_{0}=\sqrt{2} r / l, r \equiv l\left(E_{Z}-J\right) /\left(\sqrt{2} \hbar s_{t}\right), l=$ $l_{B} / \sqrt[4]{\frac{1}{4}+\frac{\omega_{0}^{2}}{\omega_{c}^{2}}}, l_{B}=\sqrt{\frac{\hbar c}{e B}}$, and $\omega_{c}=\frac{e B}{m c}$. The function $f(x)$ is introduced in the main text of the paper [see Eq. (14)], and $I_{n}(x)$ are the modified Bessel functions of the $n$-th kind. Note that the rate in Eq. (B1) has a geometrical dependence on the orientation of the setup with respect to the crystallographic axes. In Fig. 4, we have plotted this leakage rate as a function of the magnetic field for a DQD along the $x$ axis. We find that the rates are typically very small and much slower than the single-spin (and the hyperfine-induced two-spin) relaxation rates.
1 D. Loss and D.P. DiVincenzo, Phys. Rev. A 57, 120 (1998).

2 R. Hanson, L. P. Kouwenhoven, J. R. Petta, S. Tarucha, and L. M. K. Vandersypen, Rev. Mod. Phys. 79, 1217 (2007).

3 A.V. Khaetskii and Yu.V. Nazarov, Phys. Rev. B 64, 125316 (2001).

${ }^{4}$ V.N. Golovach, A.V. Khaetskii, and D. Loss, Phys. Rev. Lett. 93016601 (2004).

5 M. Borhani, V.N. Golovach, and D. Loss, Phys. Rev. B 73, 155311 (2006).

6 S. Amasha, K. MacLean, I.P. Radu, D.M. Zumbuhl, M.A. Kastner, M.P. Hanson, and A.C. Gossard, Phys. Rev. Lett. 100046803 (2008).

7 S. Shankar, A. M. Tyryshkin, J. He, and S. A. Lyon, Phys. Rev. B 82, 195323 (2010).

${ }^{8}$ H. Bluhm, S. Foletti, I. Neder, M. Rudner, D. Mahalu,
V. Umansky, and A.Yacoby, Nature Physics 7, 109 (2011).

9 R. B. Liu, W. Yao, and L. J. Sham, New J. Phys. 9, 226 (2007); L. Cywinski, W. M. Witzel, and S. Das Sarma, Phys. Rev. Lett. 102, 057601 (2009); C. Deng and X. Hu, Phys. Rev. B 78, 245301 (2008); W. A. Coish, J. Fischer, and D. Loss, Phys. Rev. B 81, 165315 (2010).

10 I. Zutic, J. Fabian, and S. Das Sarma, Rev. Mod. Phys. 76, 323 (2004).

11 E.I. Rashba and Al.L. Efros, Phys. Rev. Lett. 91, 126405 (2003); Appl. Phys. Lett. 83, 5295 (2003); C. Flindt, A. S. Sorensen, and K. Flensberg, Phys. Rev. Lett. 97, 240501 (2006); D. V. Bulaev and D. Loss, Phys. Rev. Lett. 98, 097202 (2007).

12 V.N. Golovach, M. Borhani, and D. Loss, Phys. Rev. B 74, 165319 (2006).

13 K. C. Nowack, F. H. L. Koppens, Yu. V. Nazarov, and L. 
M. K. Vandersypen, Science 318, 1430 (2007).

14 S. Nadj-Perge, S. M. Frolov, E. P. A. M. Bakkers, and L. P. Kouwenhoven, Nature 468, 1084 (2010).

15 M. D. Schroer, K. D. Petersson, M. Jung, and J. R. Petta, arXiv:1105.1462 (unpublished).

16 C. Fasth, A. Fuhrer, L. Samuelson, V. N. Golovach, and D. Loss, Phys. Rev. Lett. 98266801 (2007).

17 A. Pfund, I. Shorubalko, K. Ensslin, and R. Leturcq, Phys. Rev. B 76 161308(R) (2007).

18 G. Dresselhaus, Phys. Rev. 100, 580 (1955).

19 Y. Bychkov and E. I. Rashba, J. Phys. C 17, 6039 (1984).

20 J.R. Schrieffer, P.A. Wolff, Phys. Rev. 149, 491 (1966).

${ }^{21}$ C. E. Pryor and M. E. Flatté, Phys. Rev. Lett. 96, 026804 (2006).
22 J. R. Petta, A. C. Johnson, J. M. Taylor, E. A. Laird, A. Yacoby, M. D. Lukin, C. M. Marcus, M. P. Hanson, and A. C. Gossard, Science 309, 2180 (2005).

${ }^{23}$ K. Ono, G. Austing, Y. Tokura, and S. Tarucha, Science 297, 1313 (2002).

24 F. Baruffa, P. Stano, and J. Fabian, Phys. Rev. Lett. 104, 126401 (2010); Phys. Rev. B 82, 045311 (2010).

25 G. Mahan, Many Particle Physics (Kluwer Academic/Plenum Publishers, Berlin, New York, 2000).

26 X. Hu and S. Das Sarma, Phys. Rev. Lett. 96, 100501 (2006).

27 X. Hu, Phys. Rev. B 83, 165322 (2011). 